\title{
Metabolic Syndrome in Rural Community
}

\author{
Mehmood Alam Pasha
}

Dear Editor,

According to our observation, prevalence of metabolic syndrome is rising in urban populations as well as rural communities. However, this rising problem is largely being ignored and according to preliminary results quality adjusted life years can be increased by preventing Metabolic Syndrome. The first attempt to give a proper definition of Metabolic Syndrome was done by World Health Organization (WHO) in $1998 .{ }^{1}$ According to WHO criteria metabolic syndrome consists of diabetes mellitus along with any two of the following: BP greater than 140/90 mm Hg, hypertriglyceridemia, decreased HDL, central obesity and microalbumenemia. ${ }^{2}$ Metabolic syndrome is a major use of morbidity in diabetic patients and medical establishments.

A research was conducted in Rajan Dhall Hospital New Delhi, India. According to the research a high prevalence of metabolic syndrome has come forward not only in urban areas of Asian Indian adults but also in the population living in the slums and rural areas. Major risk factors were over nutrition, sedentary lifestyle, increased urbanization and rapid migration from rural to urban areas. ${ }^{3}$ A similar study was conducted in USA by assessing waist circumference, serum HDL, serum cholesterol and serum glucose levels and it showed that $22.8 \%$ men and $22.6 \%$ women had the syndrome. The major risk factors in the USA were old age, postmenopausal women, high BMI, smoking, low income, high carbohydrate diet and physical inactivity. ${ }^{4}$ A research conducted under NCEP (National Cholestrerol Evaluation Program) to evaluate and quantify people suffering from Coronary Heart Disease

Pasha M.A. ${ }^{1}$

Department of Community Medicine, King Edward Medical University, Lahore
(CHD) among the population suffering from Metabolic Syndrome. The study clearly showed that prevalence of CHD markedly increased with the presence of metabolic syndrome. It also showed that this ratio increased when the subjects had Diabetes along with metabolic syndrome.

Our study will outline all the risk factors including hypertension, hypertriglyceridemia, decreased HDL, central obesity and micro albuminuria which lead to prevalence of metabolic syndrome in diabetics in Maraca. It is very essential to do yearly surveys to decrease burden of non communicable diseases. The intervention through health education and awareness can help in shifting the paradigm of approach to cure this syndrome and its complications towards more realistic and holistically oriented prevention of metabolic syndrome. Moreover, various fruitful research efforts can be based on the results of this research to ascertain the risk factors. There were no conflicts of interest declared.

\section{Acknowledgements}

We would like to thank Dr. Muhammad Arif Khan of Department Community Medicine King Edward Medical University.

\section{References}

1. Alberti KG, Zimmet PZ. Definition, diagnosis and classification of diabetes mellitus and its complications. Part 1: diagnosis and classification of diabetes mellitus provisional report of a WHO consultation. Diabetic Medicine, 1998; 15: 539-553.

2. Marchesini G, Bugianesi E, Forlani G, Cerelli F, Lenzi M, Manini R, et al. Nonalcoholic fatty liver, steatohepatitis, and the metabolic syndrome. Hepatology 2003; 37: 917-923. Doi:10.1053/jhep.2003.50161 
3. Misra A, Kharana L. The metabolic syndrome in South Asians: epidemiology, determinants, and prevention. Metabolic syndrome and Related Disorders, 2009;7 (6): 497-514. Doi:10.1089/met.2009.0024.
4. Ford ES. Prevalence of the metabolic syndrome in US populations. Endocrinology and metabolism clinics of North America, 2004; 33: 333-350. 